\title{
OTIMIZAÇÂO DAS CONDIÇÕES DE PREPARAÇÃO DE ELETRODOS À BASE DE CARBONO CERÂMICO UTILIZANDO-SE PLANEJAMENTO FATORIAL
}

\author{
Tatiane Skeika e Christiana Pessoa* \\ Departamento de Química, Universidade Estadual de Ponta Grossa, 84030-900 Ponta Grossa - PR, Brasil \\ Sergio Toshio Fujiwara \\ Departamento de Química, Universidade Estadual do Centro Oeste - 85040-080 Guarapuava - PR, Brasil \\ Noemi Nagata \\ Departamento de Química, Universidade Federal do Paraná, 81531-980 Curitiba - PR, Brasil
}

Recebido em 28/4/09; aceito em 13/10/09; publicado na web em 11/3/10

\begin{abstract}
OPTIMIZATION OF ELECTRODES CONDITIONS PREPARATION BASED CARBON CERAMIC USING FACTORIAL DESIGN. Different parameters of carbon ceramic electrodes (CCE) preparation, such as type of precursor, carbon material, catalyst amount, among others, significantly influence the morphological properties and consequently their electrochemical responses. This paper describes a $2^{3}$ factorial design ( 2 factors and 3 levels with central point replicates), which the factors analyzed were catalyst amount $\left(\mathrm{HCl} 12 \mathrm{~mol} \mathrm{~L}^{-1}\right)$, graphite/precursor ratio, and precursor type (TEOS - tetraethoxysilane and MTMOS methyltrimetoxysilane). The design resulted in a significant third order interaction for peak current values (Ipa) and a second order interaction for potential difference $(\Delta \mathrm{E})$, between thefactors studied, which could not be observed when using an univariated study.
\end{abstract}

Keywords: carbon ceramic electrodes; cyclic voltammetry; factorial design.

\section{INTRODUÇÃO}

Recentemente tem havido um crescente interesse no estudo de uma nova geração de materiais que apresentam alta condutividade elétrica, os chamados eletrodos de carbono cerâmico (ECC), os quais são basicamente obtidos através da dopagem da matriz de sílica obtida pelo método sol-gel com o pó de carbono ou grafite..$^{1-5}$ Os primeiros eletrodos obtidos através dessa técnica foram propostos pelo grupo de Lev e colaboradores e desde então têm sido aplicados na determinação de diversas espécies de interesse farmacológico e ambiental. ${ }^{6}$ Existem inúmeras vantagens destes materiais em relação aos demais eletrodos à base de carbono, sendo que a principal delas consiste na combinação das propriedades do processo sol-gel (tal como alta área superficial) e a condutividade dos materiais de carbono, possibilitando a obtenção de um eletrodo de superfície renovável similar ao eletrodo de pasta de carbono, porém mais robusto e de maior estabilidade..$^{7-11}$

O método de preparação do eletrodo de carbono cerâmico influencia diretamente nas suas propriedades condutoras, mecânicas e morfológicas, sendo estas refletidas na resposta eletroquímica do eletrodo. A maior parte das diferentes configurações de eletrodos de carbono cerâmico descritas na literatura utiliza precursores hidrofóbicos, em particular o metiltrimetoxissilano, para a construção de sensores eletroquímicos. ${ }^{12-20}$ Tal característica pode ser explicada devido ao aumento da hidrofobicidade da interfase eletrodo-solução, possibilitando que somente poucas partículas de carbono na superfície do eletrodo fiquem em contato com o eletrólito. Esta configuração minimiza a corrente de fundo, a qual é proporcional à superfície condutora do eletrodo, aumentando a sensibilidade do mesmo. ${ }^{10}$ Por outro lado, a obtenção de um eletrodo compósito hidrofílico é menos empregada, permitindo a construção de um eletrodo permeável a soluções aquosas. ${ }^{15}$

O tipo de material de carbono é considerado outro fator que influencia na resposta dos ECC, em particular na sua condutividade e propriedades mecânicas. ${ }^{21-25}$ Recentemente, os eletrodos compósi-

*e-mail: christiana.pessoa@pq.cnpq.br tos têm sido preparados com diferentes tipos de materiais à base de carbono, tais como, os nanotubos de carbono, ${ }^{14,16,21}$ carbono vítreo, ${ }^{17}$ além de grafite e derivados. ${ }^{26-34}$ Por exemplo, Sun e colaboradores utilizaram o carbono vítreo na preparação do eletrodo compósito de carbono vítreo cerâmico (denominado ECCV), que apresentou uma pequena separação de potenciais de pico em meio a ferrocianeto de potássio de $\Delta \mathrm{Ep}=66 \mathrm{mV}$, similar ao do ECC preparado com grafite $(\Delta \mathrm{Ep}=60 \mathrm{mV})$. Porém, devido à estrutura particulada do carbono vítreo, o ECCV se mostrou mais versátil no caso da modificação com fenantrenoquinona, além de apresentar alta rigidez mecânica e ampla janela de potencial. ${ }^{17} \mathrm{O}$ emprego de nanotubos de carbono em eletrodos compósitos tem resultado em eletrodos de carbono cerâmico com propriedades elétricas e mecânicas aprimoradas, que favorecem sua aplicação como sensores. ${ }^{16}$

A proporção grafite/precursor e a quantidade de ácido clorídrico utilizado influenciam significativamente na organização estrutural e porosidade do material de carbono obtido. ${ }^{11}$ Um exemplo dessa influência foi descrita por Zhu e colaboradores, que estudaram uma melhor relação de proporção entre o material de carbono e o precursor (metiltrimetoxissilano), analisando as respostas eletroquímicas para os eletrodos de carbono cerâmico. Nesse estudo, os resultados mais promissores foram obtidos utilizando-se uma proporção intermediária entre a quantidade de grafite e da mistura hidrolisada (50/50\% $\mathrm{m} / \mathrm{m}$ ), na qual foi verificada uma melhor reversibilidade do sistema eletroquímico (menor resistividade), além de maior sensibilidade em meio a ferrocianeto de potássio. ${ }^{14}$

Em função dos diferentes parâmetros que influenciam as características do ECC preparado e, principalmente, dos efeitos de interação já observados por outros autores na preparação de eletrodos, a otimização da resposta do ECC utilizando-se métodos quimiométricos é de grande importância na obtenção dos mesmos. Dentre os planejamentos quimiométricos mais utilizados estão os fatoriais $2^{\mathrm{k}}$ (2 níveis e $k$ fatores), os quais permitem otimizar o sistema, com um pequeno número de experimentos e, principalmente, tornam possível a verificação dos efeitos entre os fatores nos diferentes níveis estudados. ${ }^{35-37}$ Essa metodologia foi empregada por Kubota e colaboradores 
na proposta de otimização da preparação de um eletrodo de pasta de carbono modificado com riboflavina imobilizada em suporte inorgânico, utilizando-se um planejamento fatorial do tipo $2^{3}$ (2 níveis e 3 fatores), no qual foram analisados os seguintes fatores: a relação entre as quantidades de grafite e sílica modificada (fator 1), a forma de preparação (fator 2) e a concentração de eletrólito suporte (fator 3). ${ }^{37}$

As diferentes condições de síntese influenciam de maneira significativa a resposta eletroquímica dos ECC. No entanto, apesar da síntese dos mesmos ser conhecida na literatura, a maior parte dos trabalhos acerca da otimização dos parâmetros de síntese é realizada de maneira univariada, o que não permite constatar efeitos de interação existentes entre os fatores. Neste contexto, a metodologia de planejamento fatorial $2^{3}$ foi empregada para otimizar a preparação do ECC, a partir de 2 diferentes precursores (tetraetoxissilano - TEOS e metiltrimetoxissilano - MTMOS), a fim de verificar a influência das diferentes estruturas dos mesmos sobre as respostas eletroquímicas dos ECC. Para tal, a quantidade de catalisador, a proporção grafite/ precursor e o tipo de precursor foram os fatores estudados, sendo analisadas as respostas de corrente e a variação dos potenciais de picos dos eletrodos obtidos na presença de ferrocianeto de potássio.

\section{PARTE EXPERIMENTAL}

\section{Síntese do eletrodo de carbono cerâmico e planejamento fatorial}

Primeiramente preparou-se a mistura hidrolisada (onde grupos alcóxidos do precursor são convertidos em grupos do tipo silanol), ${ }^{11}$ contendo 1,5 mL de metanol, que atua como solvente da reação, além de três quantidades distintas de ácido clorídrico $12 \mathrm{~mol} \mathrm{~L}^{-1}[15 \mu \mathrm{L}$ referente ao nível (-), $25 \mu \mathrm{L}$ para o nível (0), e $35 \mu \mathrm{L}$ ao nível (+)]. Adicionaram-se, ainda, quantidades variadas (conforme a Tabela 1) dos precursores (MTMOS ou TEOS), mantendo-se o sistema sob agitação durante $5 \mathrm{~min}$, sendo realizado um planejamento fatorial para cada um dos precursores.

Tabela 1. Proporções de grafite/precursor referentes ao nível determinado pelos ensaios realizados no processo de otimização de experimentos

\begin{tabular}{lccc}
\hline$(\% \mathrm{~m} / \mathrm{m})$ & $(-) 50 / 50$ & $(0) 55 / 45$ & $(+) 60 / 40$ \\
\hline Grafite & $1,5 \mathrm{~g}$ & $1,65 \mathrm{~g}$ & $1,8 \mathrm{~g}$ \\
Precursor * & $1,5 \mathrm{~mL}$ & $1,35 \mathrm{~mL}$ & $1,20 \mathrm{~mL}$ \\
\hline
\end{tabular}

* MTMOS: $\mathrm{d}=0,955 \mathrm{~g} / \mathrm{mL}$; TEOS: $\mathrm{d}=0,934 \mathrm{~g} / \mathrm{mL}$

A mistura obtida foi adicionada em quantidades variadas de grafite (Tabela 1), que também seguiram a proporção referente ao nível do planejamento. Após esta etapa, introduziu-se o sol resultante em um tubo de vidro (área exposta de aproximadamente 0,2 $\mathrm{cm}^{2}$ ), inserindo-se um fio condutor de níquel-crômio (Ni-Cr), para o contato elétrico. Os eletrodos obtidos foram submetidos ao processo de secagem à temperatura ambiente durante o período de 1 semana.

Foi realizado um planejamento fatorial $2^{3}$, analisando-se a interação entre as diferentes condições de preparação e as propriedades eletroquímicas dos ECC. Os fatores estudados foram a proporção grafite/precursor, a quantidade de $\mathrm{HCl}$ e o tipo de precursor onde os valores utilizados foram variados de acordo com o intervalo apresentado na Tabela 2.

Após as diferentes sínteses variando-se os fatores nos diferentes níveis (Tabela 2), as respostas eletroquímicas dos ECC foram analisadas com relação às correntes de pico anódica (Ipa) e a variação de potenciais $(\Delta \mathrm{E})$ dos respectivos voltamogramas cíclicos. Foram calculados os efeitos principais e os efeitos de interação (segunda e terceira ordem) referentes às respostas estudadas, observando-se a presença ou não de efeitos significativos.
Tabela 2. Coeficientes de contraste para o planejamento fatorial $2^{3} \mathrm{com}$ replicatas do ponto central e respostas eletroquímicas

\begin{tabular}{|c|c|c|c|c|c|}
\hline \multicolumn{3}{|l|}{ FATORES } & $(-)$ & $(0)$ & $(+)$ \\
\hline \multicolumn{3}{|c|}{ 1) $\mathrm{HCl}(\mu \mathrm{L})$} & 15 & 25 & 35 \\
\hline \multicolumn{3}{|c|}{ 2) Grafite/precursor $(\% \mathrm{~m} / \mathrm{m})$} & $50 / 50$ & $55 / 55$ & $60 / 40$ \\
\hline \multicolumn{3}{|c|}{ 3) Tipo Precursor } & MTMOS & - & TEOS \\
\hline ENSAIO & $\mathrm{HCl}$ & Proporção & Tipo & $\operatorname{Ipa}(\mu \mathrm{A})$ & $\Delta \mathrm{E}(\mathrm{mV})$ \\
\hline 1 & - & - & - & 5,83 & 198,8 \\
\hline 2 & + & - & - & 9,61 & 234,6 \\
\hline 3 & - & + & - & 10,34 & 165,7 \\
\hline 4 & + & + & - & 0,22 & 389,6 \\
\hline 5 & - & - & + & 0,33 & 447,5 \\
\hline 6 & + & - & + & 0,25 & 447,2 \\
\hline 7 & - & + & + & 1,13 & 370,7 \\
\hline 8 & + & + & + & 0,32 & 408,0 \\
\hline 9 & 0 & 0 & - & 6,22 & 96,7 \\
\hline 10 & 0 & 0 & - & 3,73 & 88,1 \\
\hline 11 & 0 & 0 & - & 6,93 & 71,7 \\
\hline 12 & 0 & 0 & + & 0,44 & 475,7 \\
\hline 13 & 0 & 0 & + & 0,29 & 408,1 \\
\hline 14 & 0 & 0 & + & 0,33 & 415,2 \\
\hline
\end{tabular}

\section{Estudos eletroquímicos}

As medidas eletroquímicas foram realizadas em um potenciostato OMNI - 101, conectado a um microcomputador para a aquisição dos dados. Foi utilizado um sistema convencional de três eletrodos: contraeletrodo de platina, eletrodo de referência $(\mathrm{Ag} / \mathrm{AgCl})$ e o ECC, como eletrodo de trabalho.

As respostas eletroquímicas de potenciais e correntes de pico foram obtidas pela técnica de voltametria cíclica, na presença de 10 $\mathrm{mL}$ de solução de ferrocianeto de potássio $\left(1,0 \mathrm{mmol} \mathrm{L}^{-1}\right)$ em meio ao eletrólito suporte $\left(\mathrm{NaCl} 0,5 \mathrm{~mol} \mathrm{~L}^{-1}, \mathrm{pH}=7\right)$, contidos na célula eletroquímica. A faixa de potencial estudada foi de -100 a $700 \mathrm{mV}$ e velocidade de varredura de $50 \mathrm{mVs}^{-1}$, para os estudos de otimização, sendo esta variada de 10 a $100 \mathrm{mVs}^{-1}$ nos estudos de caracterização eletroquímica do ECC otimizado.

\section{Caracterização morfológica}

A caracterização morfológica dos ECC foi realizada a partir da técnica de microscopia eletrônica de varredura (MEV), utilizandose um equipamento Shimadzu SSX-550. As amostras foram fixadas no porta-amostra utilizando-se uma fita dupla-face condutora de grafite, sem a necessidade de tratamento prévio, já que o carbono cerâmico também possui propriedades condutoras. As micrografias dos ECC preparados foram obtidas com um aumento de 1500 vezes, na escala de $10 \mu \mathrm{m}$

\section{RESULTADOS E DISCUSSÃO}

\section{Síntese do eletrodo de carbono cerâmico}

O processo sol-gel na obtenção dos eletrodos de carbono cerâmico pode ser explicado pela reação de hidrólise em meio ácido do grupo alcóxido com a formação de grupos reativos do tipo silanol, seguida da etapa da condensação do grupo silanol, ambas ocorridas via substituição nucleofílica bimolecular no átomo de silício. ${ }^{11}$ Abaixo estão mostradas as reações de hidrólise dos diferentes precursores com subsequente condensação dos grupos silanóis em meio ácido: 
Reação de hidrólise do MTMOS

$\left.\mathrm{CH}_{3} \mathrm{Si}\left(\mathrm{OCH}_{3}\right)_{3}+\mathrm{H}_{2} \mathrm{O} \mathrm{CH} \mathrm{CH}_{3} \mathrm{SiCH}\right)_{2} \rightarrow(\mathrm{OH})+\mathrm{CH}_{3} \mathrm{OH}$

Reação de hidrólise do TEOS

$\mathrm{Si}\left(\mathrm{OCH}_{2} \mathrm{CH}_{3}\right)_{4}+2 \mathrm{H}_{2} \mathrm{O} \mathrm{Si}\left(\mathrm{OCH}_{2} \mathrm{CH}_{3}\right)_{2} \rightarrow(\mathrm{OH})_{2}+2 \mathrm{CH}_{2} \mathrm{CH}_{3} \mathrm{OH}$

Condensação dos grupos silanóis em meio ácido:

$=\mathrm{Si}-\mathrm{OH}+\mathrm{HO}-\mathrm{Si}=\rightarrow=\mathrm{Si}-\mathrm{O}-\mathrm{Si}=+\mathrm{H}_{2} \mathrm{O}$

A dopagem do esqueleto de sílica formado pela condensação dos grupos silanóis ocorre na etapa posterior à síntese. Quando o material de carbono é adicionado sobre a mistura hidrolisada, as partículas de grafite se arranjam entre os esqueletos da matriz de sílica e, após a evaporação do solvente. o carbono cerâmico é obtido. ${ }^{11}$

As características dos eletrodos de carbono cerâmico estão diretamente relacionadas à estrutura do precursor utilizado na obtenção do compósito. Um exemplo dessa influência se reflete na diminuição das correntes de fundo de eletrodos compósitos, devido ao aumento da hidrofobicidade do precursor. ${ }^{10}$ Em função disto, a escolha dos precursores MTMOS e TEOS para a obtenção dos ECC foi baseada na hidrofobicidade destes permitindo, dessa forma, verificar a influência das diferentes estruturas dos precursores nas propriedades do ECC.

Além disso, as propriedades físicas (tais como, área superficial específica, tamanho médio dos poros) de um gel seco (o xerogel) dependem fortemente dos parâmetros utilizados na obtenção do processo de sol-gel, fato que motivou o estudo de planejamento e otimização das condições de preparação do ECC.

\section{Planejamento fatorial}

A maior parte dos trabalhos publicados recentemente na área de eletrodos de carbono cerâmico avalia individualmente a influência de alguns parâmetros na preparação desses eletrodos, levando-se em consideração suas propriedades eletroquímicas. ${ }^{11,14-17}$ Dessa forma, um estudo para a análise do comportamento eletroquímico de ECC obtidos a partir de dois tipos de precursores, TEOS e MTMOS, foi realizado empregando-se a metodologia de planejamento fatorial. Além de se avaliar a influência de cada variável sobre as respostas dos eletrodos, foi possível verificar a interação entre elas. As variáveis estudadas foram a quantidade de $\mathrm{HCl}$ concentrado do processo sol-gel, a proporção grafite/precursor e o tipo de precursor. Como esta última variável estudada é qualitativa, o planejamento fatorial completo com ponto central foi construído realizando-se uma triplicata com o precursor MTMOS e outra com o TEOS.

As respostas do ECC foram analisadas por voltametria cíclica na presença de ferrocianeto de potássio (utilizado como molécula sonda). Os resultados voltamétricos obtidos para os eletrodos preparados nas diferentes condições estão mostrados na Tabela 2. A determinação da estimativa do desvio padrão foi realizada a partir das replicatas do ponto central, a fim de verificar os efeitos significativos de cada variável sobre as respostas dos eletrodos.

Analisando-se os resultados obtidos, uma maior sensibilidade de corrente $(\mathrm{Ipa}=10,34 \mu \mathrm{A})$ e reversibilidade $(\Delta \mathrm{E}=165,70 \mathrm{mV})$ na resposta eletroquímica foi obtida para o ECC preparado com precursor MTMOS, $15 \mu \mathrm{L}$ de $\mathrm{HCl}$ e proporção 60/40\% m/m (grafite/precursor). No entanto, pode ser constatado que os dados eletroquímicos referentes ao ponto central preparado com o precursor MTMOS, quando comparados ao ensaio 3 , resultaram em menores valores de $\Delta \mathrm{E}$. Por outro lado, essa mesma triplicata apresentou uma menor sensibilidade, além do deslocamento de potenciais de pico para regiões mais positivas $(\mathrm{Epa}=476,5 \mathrm{mV}, \mathrm{Epc}=404,8 \mathrm{mV})$, em relação ao ensaio 3 $(\mathrm{Epa}=334,6 \mathrm{mV}, \mathrm{Epc}=168,9 \mathrm{mV})$, sendo este último mais propício no caso de aplicação como sensor eletroquímico.
O tratamento estatístico evidenciou que os fatores que obtiveram efeito significativo (com 95\% de confiança) para os valores de Ipa foram o tipo de precursor, a interação entre quantidade de catalisador e a proporção grafite/precursor, assim como a interação entre os três fatores analisados (Figura 1A). Essa interação de terceira ordem indica que para a obtenção de uma melhor sensibilidade de corrente os três fatores estudados devem ser levados em consideração.

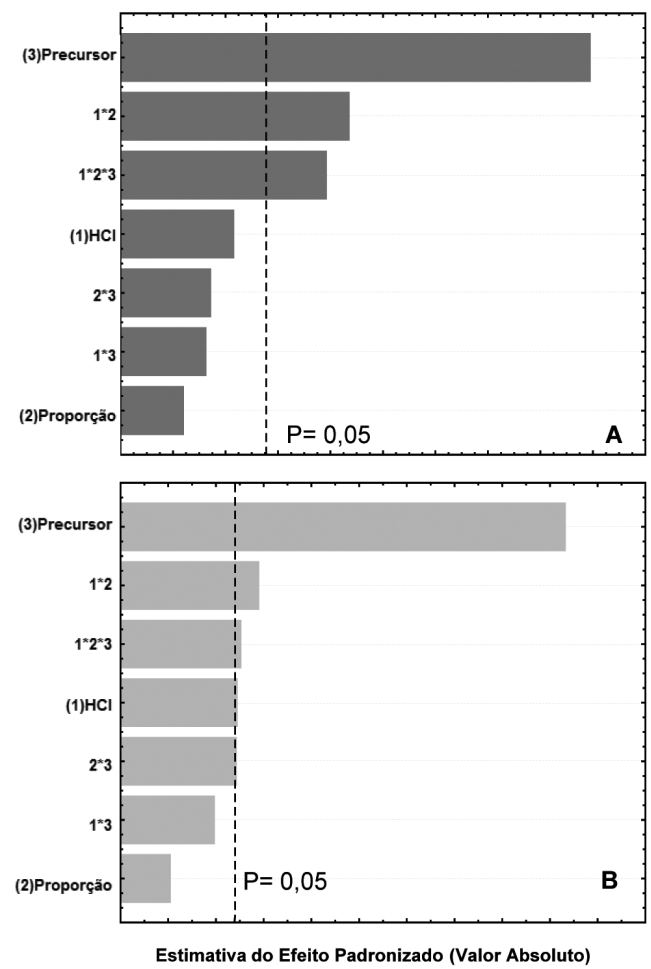

Figura 1. Diagrama de Pareto para os efeitos padronizados considerando como resposta (A) Ipa ( $\mu A) ;(B) \Delta E(m V)$

Todos esses efeitos podem ser melhor visualizados através da interpretação geométrica dos dados (Figura 2). O efeito principal 3 (tipo de precursor) para os valores de Ipa pode ser visualizado na face correspondente ao precursor MTMOS (nível -), três dos quatro vértices possuem valores de sensibilidade de corrente maiores quando comparadas aos valores da face referente ao TEOS (apenas o vértice +++ é mais sensível comparado à face do MTMOS). O efeito de interação entre os fatores 1 x 2 (face do cubo em destaque) pode ser observado fixando-se a quantidade de $\mathrm{HCl}$ e o tipo precursor (níveis - -), um acréscimo de 4,51 $\mu \mathrm{A}$ na sensibilidade de Ipa ocorre quando se varia a proporção do menor para o maior nível. Porém, fixando-se o tipo de precursor (nível -) na quantidade de $\mathrm{HCl}$ no nível (+) a tendência de acréscimo de corrente não foi observada; pelo contrário, houve um decréscimo de 9,39 $\mu \mathrm{A}$, evidenciando que o efeito da quantidade de $\mathrm{HCl}$ depende da proporção grafite/precursor utilizada. Finalmente, o efeito de interação $1 \times 2 \times 3(3,29 \mu \mathrm{A}$, Tabela 3$)$ pode ser observado através da comparação entre os vértices opostos (- + -, ensaio 3$)$ e o (+ - +, ensaio 5). Verifica-se que com a variação dos três fatores ao mesmo tempo, houve uma grande variação nos valores de Ipa $(10,09 \mu \mathrm{A})$. Nenhuma outra comparação entre vértices opostos gera um valor dessa magnitude, confirmando que a interação entre os três fatores influencia diretamente sobre a resposta de corrente do ECC.

A partir do cálculo dos efeitos observou-se que para os valores de $\Delta \mathrm{E}$ todas as interações de segunda ordem são significativas (Figura 1B), o que pode ser melhor visualizado pela interpretação geométrica para os dados de variação de potencial (Figura 3). Neste caso, é pos- 
sível visualizar a face correspondente ao precursor MTMOS (nível -), sendo que os quatro vértices possuem valores menores de $\Delta \mathrm{E}$ quando comparados aos da face referente ao TEOS. Analisando-se a face com melhor resultado (em termos de $\Delta \mathrm{E}$ ) foi possível observar o efeito de interação $1 \times 2$ (56,4 mV, Tabela 3), cuja interpretação indica um aumento na reversibilidade (diminuição do $\Delta \mathrm{E}$ ) analisando-se o fator $\mathrm{HCl}$ fixo no nível (-) e variando-se o fator proporção. Ao contrário, uma diminuição de reversibilidade (representada pelo aumento de $155,00 \mathrm{mV}$ ) foi observada quando se fixou o fator $\mathrm{HCl}$ no nível (+).

É importante salientar que uma baixa reprodutibilidade foi observada nas triplicatas dos pontos centrais (estimativa de desvio padrão - Ipa: $1,68 \mu \mathrm{A}$ e $\Delta \mathrm{E}: 12,7 \mathrm{mV}$ ), provavelmente em função de dificuldades no controle de algumas variáveis associadas à construção do eletrodo (p. ex. empacotamento da matriz de carbono cerâmico). Entretanto, é necessário esclarecer que a realização de múltiplas medidas com um único eletrodo fornece uma excelente repetibilidade (ver resultados no item Caracterização eletroquímica do ECC otimizado), o que, pelo menos em princípio, sugere uma boa potencialidade analítica.

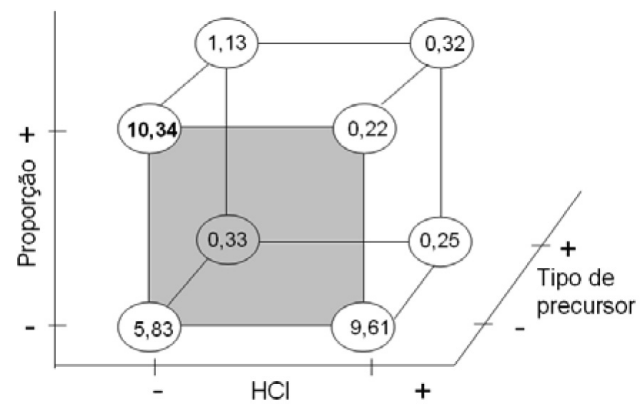

Figura 2. Interpretação geométrica dos efeitos do planejamento fatorial $2^{3}$ considerando os valores de Ipa

Tabela 3. Efeitos calculados para um planejamento fatorial $2^{3}$

\begin{tabular}{|c|c|c|c|}
\hline Ipa $(\mu \mathrm{A})$ & & $\Delta \mathrm{E}(\mathrm{mV}$ & \\
\hline Média: $3,28 \mu \mathrm{A} \pm 0,3$ & & Média: $301,2 \mathrm{~m}$ & $V \pm 7,42$ \\
\hline Efeitos Principais & & Efeitos Prin & cipais \\
\hline 1) $\mathrm{HCl}$ & $-1,81 \pm 0,84$ & 1) $\mathrm{HCl}$ & $74,2 \pm 19,6$ \\
\hline 2) Grafite/precursor & $-1,00 \pm 0,84$ & 2) Grafite/precursor & $1,47 \pm 19,6$ \\
\hline 3) Tipo de precursor & $-5,68 \pm 0,64$ & 3) Tipo de precursor & $246,7 \pm 14,8$ \\
\hline Interação de $2^{\underline{a}}$ ordem & & Interação de 2 & ordem \\
\hline $1 \times 2$ & $-3,66 \pm 0,84$ & $1 \times 2$ & $56,4 \pm 19,6$ \\
\hline $1 \times 3$ & $1,36 \pm 0,84$ & $1 \times 3$ & $-55,7 \pm 19,6$ \\
\hline $2 \times 3$ & $1,44 \pm 0,84$ & $2 \times 3$ & $-59,5 \pm 19,6$ \\
\hline Interação de $3^{\underline{a}}$ ordem & & Interação de 3 & ordem \\
\hline $1 \times 2 \times 3$ & $3,29 \pm 0,84$ & $1 \times 2 \times 3$ & $-37,6 \pm 19,6$ \\
\hline
\end{tabular}

valores em negrito $=$ efeitos estatisticamente significativos com $95 \%$ de confiança.

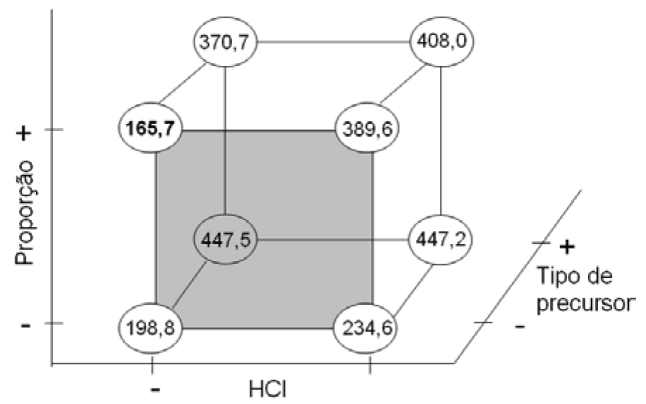

Figura 3. Interpretação geométrica dos efeitos do planejamento fatorial $2^{3}$ considerando os valores de $\Delta E$
Comparando-se os valores de Ipa e $\Delta \mathrm{E}$ obtidos através do planejamento fatorial conclui-se que as melhores condições para obtenção dos ECC seriam referentes ao ensaio $3[15 \mu \mathrm{L}$ de $\mathrm{HCl}$, proporção $60 / 40 \mathrm{~m} / \mathrm{m}$ (grafite/precursor) e precursor MTMOS]. A maioria dos trabalhos publicados utilizam $50 \mu \mathrm{L}$ de $\mathrm{HCl}$ e a proporção $50 / 50 \mathrm{~m} / \mathrm{m}$ e o MTMOS como precursor. ${ }^{1,4,7,8,12}$ Porém, a diminuição da quantidade de $\mathrm{HCl},{ }^{14,15}$ e a variação da proporção ${ }^{14,16}$ pode conferir ao ECC propriedades eletroquímicas diferenciadas. ${ }^{11}$ A utilização de menor quantidade de $\mathrm{HCl}$ pode resultar em um material cerâmico mais poroso, o que favorece a difusão das espécies do eletrólito. Por outro lado, a maior proporção de grafite em relação ao precursor pode proporcionar um aumento na condutividade do ECC. Analisando-se os voltamogramas dos eletrodos nestas condições observa-se maior sensibilidade de corrente para o ECC sintetizado com MTMOS, além de maior reversibilidade do sistema (Figura 4A), em relação ao obtido com TEOS nas mesmas condições (Figura 4B). Essa maior reversibilidade ( $165,7 \mathrm{mV})$ é uma indicação que o processo de transferência de elétrons ocorre de maneira mais efetiva para o eletrodo preparado com precursor MTMOS.
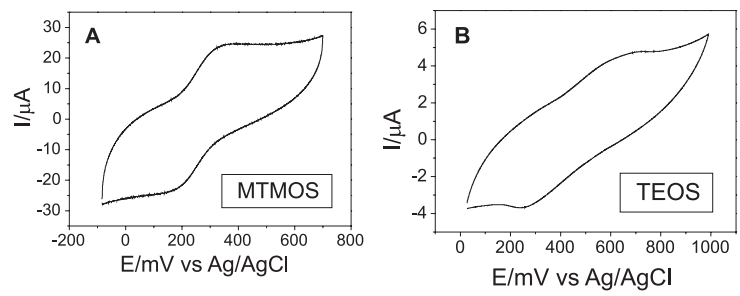

Figura 4. Voltamogramas cíclicos dos ECC obtidos nas melhores condições (15 $\mu \mathrm{L}$ de $\mathrm{HCl}$, e proporção $60 / 40 \mathrm{~m} / \mathrm{m}$ ) de preparação em meio a ferrocia-

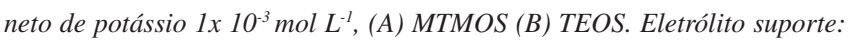
$[\mathrm{NaCl}]=0,5 \mathrm{~mol} \mathrm{~L} \mathrm{~L}^{-1}, \mathrm{v}=50 \mathrm{mV} \mathrm{s}^{-1}$

\section{Caracterização morfológica}

A caracterização morfológica dos ECC foi realizada empregando-se a técnica de microscopia eletrônica de varredura (MEV). Para facilitar as comparações e gerar indícios que possibilitassem explicar as diferentes respostas voltamétricas com os precursores MTMOS e TEOS, foram obtidas as microscopias para os melhores resultados eletroquímicos. A imagem da amostra referente ao ensaio 3 do planejamento fatorial [15 $\mu \mathrm{L}$ de $\mathrm{HCl}$, proporção 60/40 m/m (grafite/precursor) e MTMOS], indicou menor compactação dos materiais compósitos obtidos, quando comparada ao ECC do ensaio 7 [ $15 \mu \mathrm{L}$ de $\mathrm{HCl}$, proporção $60 / 40 \mathrm{~m} / \mathrm{m}$ (grafite/precursor) e TEOS]. (Figuras 5A e B, respectivamente).

Relacionando-se as imagens de MEV obtidas com a resposta eletroquímica dos mesmos (Figura 4) sugere-se que uma estrutura morfológica menos compactada do ECC, provavelmente, facilita o processo de transferência de elétrons na interfase eletrodo-solução, proporcionando uma maior reversibilidade e melhor definição dos picos voltamétricos.

\section{Caracterização eletroquímica do ECC otimizado}

Estipuladas as melhores condições de preparação do ECC, foram realizados estudos de voltametria cíclica em diferentes velocidades de varredura, resultando em uma relação linear entre os valores de corrente de pico anódico e a raiz quadrada da velocidade (Figura não mostrada). Tal resultado indica que o processo de transferência de elétrons na interfase eletrodo-solução é controlado apenas pela difusão das espécies, o que possibilita a sua aplicação como sensor eletroquímico. Ressalta-se ainda que o ECC não apresentou deslocamento de potenciais de pico com a velocidade, sugerindo que o processo redox na superfície do eletrodo ocorre de maneira eficiente. ${ }^{38}$ 

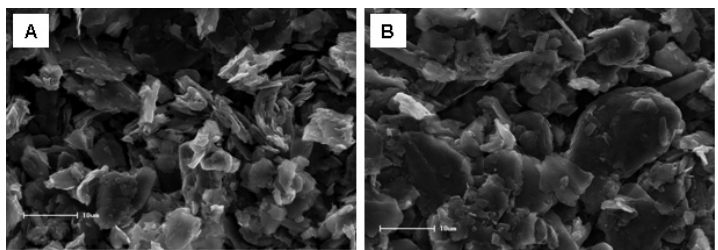

Figura 5. Imagens de MEV para os ECC preparados com $15 \mu \mathrm{L}$ de $\mathrm{HCl}$, e proporção $60 / 40 \mathrm{~m} / \mathrm{m}$ (grafite/precursor) (A) MTMOS (B) TEOS [aumento de 1500X, escala $=10 \mu \mathrm{m}]$

Para verificar a repetibilidade da resposta eletroquímica do ECC otimizado, foram obtidos voltamogramas cíclicos consecutivos do eletrodo na presença de uma concentração fixa de $\mathrm{K}_{4}\left[\mathrm{Fe}(\mathrm{CN}){ }_{6}\right](1,0$ mmol. $\left.\mathrm{L}^{-1}\right)$, numa velocidade de $50 \mathrm{mVs}^{-1}$. O perfil voltamétrico obtido foi avaliado em função dos valores de corrente de pico anódica e do potencial de pico anódico (Figura 6). Os resultados indicaram que mesmo após a aquisição de 100 ciclos de varredura não houve variações significativas tanto de Epa (Figura 6A) quanto de Ipa (Figura 6B) quando comparados aos voltamogramas inicialmente obtidos, sendo este outro fator preponderante que favorece a aplicação do ECC como sensor eletroquímico.
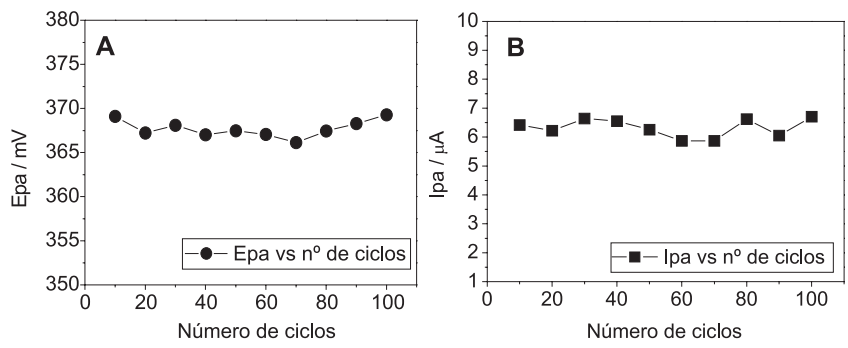

Figura 6. Estudo da repetibilidade do ECC em meio a $\mathrm{K}_{4}\left[\mathrm{Fe}(\mathrm{CN})_{6}\right]=1 \times 10^{-3}$ mol L $L^{-1}$. Condições: eletrólito suporte: $[\mathrm{NaCl}]=0,5 \mathrm{~mol} \mathrm{~L}^{-1}$ e $\mathrm{v}=50 \mathrm{mV} \mathrm{s}^{-1}$. Variação em função do número de ciclos (A) Epa (B) Ipa

\section{CONCLUSÃO}

A partir da análise do planejamento fatorial pode-se constatar maior sensibilidade e reversibilidade para o ECC preparado com 15 $\mu \mathrm{L}$ de $\mathrm{HCl}$, proporção 60/40 m/m (grafite/precursor) e MTMOS como precursor. $\mathrm{O}$ tratamento estatístico evidenciou uma interação de terceira ordem para os valores de correntes de pico indicando que, para a obtenção de melhor sensibilidade de corrente, os três fatores estudados devem ser levados em consideração. Para os valores de $\Delta \mathrm{E}$ todas as interações de segunda ordem são significativas. Ressalta-se que tais resultados não seriam observados aplicando-se um estudo univariado.

As análises de MEV dos ECC obtidas utilizando-se os diferentes precursores indicaram que provavelmente uma menor compactação facilita o processo de transferência de elétrons na interfase eletrodo-solução. $\mathrm{O}$ ECC preparado nas melhores condições estipuladas pelo planejamento fatorial apresentou uma relação linear entre corrente de pico anódica e raiz quadrada da velocidade de varredura, além de uma considerável repetibilidade, possibilitando sua aplicação como possível sensor eletroquímico. A metodologia de planejamento fatorial pode ser empregada na análise de outros parâmetros, tais como, o tipo de material de carbono e diferentes precursores, buscando otimizar ainda mais as respostas dos ECC, visando o aumento da gama de aplicação dos mesmos.

\section{REFERÊNCIAS}

1. Thenmozhi, K.; Narayanan, S. S.; Anal. Bioanal. Chem. 2007, 387, 1075 .

2. Rozniecka, E.; Shul, G.; Sirieix-Plenet, J.; Gaillon, L.; Opallo, M.; Electrochem. Commun. 2005, 7, 299.

3. Sheng, Q.; Yu, H.; Zheng, J.; J. Solid State Electrochem. 2008, 12, 1077.

4. Sheng, Q.; Yu, H.; Zheng, J.; J. Electroanal Chem. 2007, 606, 39.

5. Salimi, A.; Mamkhezri, H.; Hallaj, R.; Talanta 2007, 70, 823.

6. Tsionsky, M.; Gun, G.; Giezer, V.; Lev, O.; Anal. Chem. 1994, 66, 1747.

7. Lei, C.; Yang, Y.; Wang, H.; Shen, G.; Yu, R.; Anal. Chem. Acta 2004, 513, 379.

8. Wang, P.; Yuan, Y.; Jing, X.; Zhu, G.; Talanta 2001, 53, 863.

9. Leia, C.; Huc, S.; Gaoa, N.; Shena, G.; Yua, R.; Bioelectrochemistry 2004, 65, 33.

10. Tsionsky, M.; Lev, O.; Anal. Chem. 1995, 67, 2409.

11. Alfaya, A. S.; Kubota, L. T.; Quim. Nova 2002, 25, 835.

12. Tian, F.; Zhu, G.; Anal. Chim. Acta 2002, 451, 251.

13. Stozhko, N. H.; Morosanova, E. I.; Kolyadina, L. I.; Fomina, S. V.; J. Anal. Chem. 2007, 61, 170.

14. Zhu, L.; Tian, C.; Zhai, J.; Yang, R.; Sens. Actuators B 2007, 125, 254.

15. Macdonald, S. M.; Szot, K.; Niedziolka, J.; Marken, F.; Opallo, M.; J. Solid State Electrochem. 2008, 12, 287.

16. Gong, K.; Zhang, M.; Yan, Y.; Su, L.; Mao, L.; Xiong, S.; Chen, Y.; Anal. Chem. 2004, 76, 6500.

17. Sun, D.; Zhu, L.; Zhu, G.; Anal. Chim Acta 2007, 564, 243.

18. Cubillana-Aguilera, L. M. A.; Palacios-Santander, J.; NaranjoRodriguez, I.; Hidalgo-Hidalgo-De-Cisneros, J. L.; J. Sol-Gel Sci. Technol. 2006, 40, 55 .

19. Sheng, Q.; H. Yu, H.; Zheng, J.; Electrochim. Acta 2007, 52, 7300.

20. Salimi, A.; Roushani, M.; Haghighi, B.; Soltanian, S.; Biosens Bioelectron. 2006, 22, 220.

21. Chen, H.; Dong, S.; Biosens. Bioelectron, 2007, 22, 1811.

22. Salimi, A.; Abdi, K. ; Talanta 2007, 63, 475.

23. Salimi, A.; Roushani, M.; Hallaj, R.; Electrochim. Acta 2006, 51, 1952.

24. Opallo, M.; Saczek-Maj, M.; Shul, G.; Hayman, C. M.; Bulman Page, P. C.; Marken, F.; Electrochim. Acta 2005, 50, 1711.

25. Salimi, A.; Hallaj, R.; Ghadermazi, M.; Talanta 2005, 65, 888.

26. Nogala, W.; Rozniecka, E.; Rogalski, J.; Opallo, M.; J. Electroanal. Chem. 2006, 608, 31.

27. Cardoso, W. S.; Francisco, M. S.; Lucho, A. M. S.; Gushikem, Y.; Solid State Ionics 2004, 167, 165.

28. Maroneze, C. M.; Arenas, L. T.; Luz, R. C. S.; Benvenutti, E. V.; Landers, R.; Gushikem, Y.; Electrochim. Acta 2008, 53, 4167.

29. Razmi, H.; Heidari, H.; Habibi, E.; J. Solid State Electrochem. 2008, 12, 1579.

30. Ballarin, B.; Cassani, M. C.; Mazzoni, R.; Scavetta, E.; Tonelli, D.; Biosens. Bioelectron. 2007, 22, 1317.

31. Tan, X.; Tian, Y.; Cai, Z. X.; Anal. Bioanal. Chem. 2005, 381, 500

32. Salimi, A.; Pourbeyram, P.; Talanta 2003, 60, 205.

33. Cardoso, W. S.; Gushikem, Y.; J. Electroanal. Chem. 2005, 583, 300.

34. Zheng, J.; Sheng, Q.; Li, L.; Shen, Y.; J. Electroanal. Chem. 2007, 611, 155.

35. Ribeiro, E. S.; Dias, S. L. P.; Fujiwara, S. T.; Gushikem, Y.; Bruns, R. E.; J. Appl. Electrochem. 2003, 33, 1069.

36. Rocha, R. F.; Rosatto, S. S.; Bruns, R. E.; Kubota, L. T.; J. Electroanal. Chem. 2007, 433, 73.

37. Pereira, A. C.; Kubota, L. T.; Quim. Nova 2004, 27, 725.

38. Bard, A. J.; Faulkner, L. R.; Electrochemical Methods, Fundamentals and Applications, Wiley: New York, 1980. 